\title{
Solving the Half-Infinite Potential Well Problem via the Application of Matrix Method
}

\author{
Rohit Gupta $^{* 1}$, Rahul Gupta ${ }^{2}$ \\ ${ }^{1,2}$ Lecturer of Physics, Department of Applied Sciences, Yogananda College of Engineering and Technology, Jammu
}

\begin{abstract}
This paper adds up how the matrix method can be used for solving the one-dimensional time-independent Schrodinger's equation for some specific potential energy variation like half-infinite potential well. The matrix method is illustrated to obtain solution of the time-independent Schrodinger's equation for half-infinite potential well, which is generally done by ordinary algebraic and analytical methods. The transcendental equation determining the discrete eigenvalues for bound state and the corresponding eigenwave functions are obtained by the time-independent Schrodinger's equation for half-infinite potential well via the application of matrix method.
\end{abstract}

Keywords - Matrix Method; Schrodinger's Equation; Half-Infinite Potential Well.

\section{Introduction}

Quantum mechanics has been perceived as an essential constituent in the modules of Physics, Chemistry and Electrical Engineering [1]. It deals with the quantization of various entities especially energy and needed to deal with the submicroscopic particles [2].The applications of timeindependent Schrodinger's equation are generally analyzed by different algebraic and analytical methods [1], [2], [3]. The matrix method has been successfully implemented in science and engineering problems [4], [5], [6], [7], [8], [9], [10], [11], [12]. This paper puts forward the Matrix Method for analyzing one of the applications of Schrodinger's equation to half-infinite potential well problem. As we know for a square matrix B [5], [6], [7], [8] of order $\mathrm{n}$ with elements $a_{i j}$, we get a column matrix $Z$ and a constant $\lambda$ such that $\mathrm{BZ}=\lambda \mathrm{Z}$ or $|B-\lambda I| \mathrm{Z}=0$. This represents a matrix equation which results in $\mathrm{n}$ homogeneous linear equations [9], [10], [11], [12] having a non-trivial solution only if $|B-\lambda I|=0$. On expand $|B-\lambda I|$, we will get $\mathrm{n}^{\text {th }}$ degree equation in $\lambda$, known as the characteristic equation of $\mathrm{B}$, whose roots i.e. $\lambda_{i}$ (where $i=1,2,3, \ldots \ldots n$ ) are called Eigenvalues and corresponding to each Eigenvalue there is a non-zero solution $Z=\left[\begin{array}{c}z_{1} \\ z_{2} \\ \ldots \\ z_{n}\end{array}\right]$ known as Eigenvector [4-12]. This paper discusses the application of matrix method for solving half-infinite potential well problem to find the transcendental equation determining the discrete eigenvalues for bound state and the corresponding eigenwave functions, which is generally done by ordinary algebraic and analytical methods [1-3]. To define halfinfinite potential, considering a potential energy variation [1] as follows,

$$
\mathrm{V}(\mathrm{y})=\left[\begin{array}{c}
\infty \text { in the region } \mathrm{y}<0 \\
0 \text { in the region } y=0 \text { to } y=L \\
\boldsymbol{V}_{\mathbf{0}} \text { in the region } y>L
\end{array}\right.
$$

Such potential energy variation is known as half-infinite potential well.

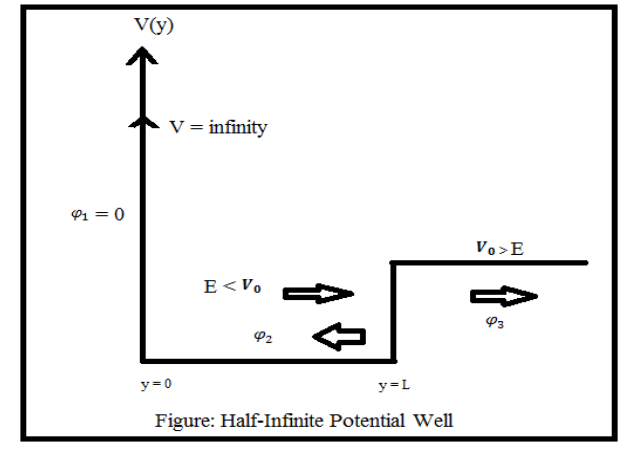

Fig.1: Half-infinite potential well

\section{Material and Method}

The one-dimensional time-independent Schrodinger's equation [3] is written as

$$
\varphi^{\prime \prime}(\mathrm{y})+\frac{8 \pi^{2} \mathrm{~m}}{\mathrm{~h}^{2}}\{\mathrm{E}-\mathrm{V}\} \varphi(\mathrm{y})=0
$$

Let $\varphi_{1}(\mathrm{y}), \varphi_{2}(\mathrm{y})$ and $\varphi_{3}(\mathrm{y})$ are the wave functions in the regions $\mathrm{y}<0,0<\mathrm{y}<\mathrm{L}$ and $\mathbf{y}>\mathrm{L}$ respectively.

Since the potential energy function $\mathrm{V}$ is infinite for $\mathrm{y}<0$, therefore from (1),

$$
\varphi_{1}(\mathrm{y})=0 \text {, for } \mathrm{y}<0 .
$$

Since the wave function is continuous at the boundaries [4], therefore, we can write

$\varphi_{1}(0)=\varphi_{2}(0)=0$

$\varphi_{3}(0)=\mathrm{a}($ say $)$

$\varphi_{2}{ }^{\prime}(0)=\varphi_{3}{ }^{\prime}(0)=\mathrm{b}$ (say)

$\varphi_{2}(\mathrm{~L})=\varphi_{3^{\prime}}(\mathrm{L})$

$\varphi_{2}(\mathrm{~L})=\varphi_{3}(\mathrm{~L})$

where $\mathrm{a}$ and $\mathrm{b}$ are constants. 
In the regions, $0<\mathrm{y}<\mathrm{L}$ and $\mathbf{y}>\mathbf{L}$, (1) can be written as

$\varphi_{2}{ }^{\prime \prime}(\mathrm{y})+k_{1}{ }^{2} \varphi_{2}(\mathrm{y})=0$

$\varphi_{3}{ }^{\prime \prime}(\mathrm{y})-{k_{2}}^{2} \varphi_{3}(\mathrm{y})=0$

where $k_{1}=\sqrt{\frac{8 \pi^{2} \mathrm{mE}}{\mathrm{h}^{2}}}$ and $k_{2}=\sqrt{\frac{2 \pi^{2} \mathrm{~m}\left(V_{0}-\mathrm{E}\right)}{\mathrm{h}^{2}}}$

To solve (2) by Matrix method [5-7],

Let $\varphi_{2}(y)=\varphi_{21}(y)$

And

$\varphi_{21}{ }^{\prime}(y)=\varphi_{22}(y) \ldots(4)$

We can rewrite (2) as

$\varphi_{22}{ }^{\prime}(y)+k_{1}^{2} \varphi_{21}(y)=0$

Or

$\varphi_{22}{ }^{\prime}(y)=-k_{1}{ }^{2} \varphi_{21}(y) \ldots \ldots(5)$

Equations (4) and (5) can be written in matrix form as $\left[\begin{array}{l}\varphi_{21}{ }^{\prime}(y) \\ \varphi_{22}{ }^{\prime}(y)\end{array}\right]=\left[\begin{array}{cc}0 & 1 \\ -k_{1}{ }^{2} & 0\end{array}\right]\left[\begin{array}{l}\varphi_{21}(y) \\ \varphi_{22}(y)\end{array}\right]$

The characteristic equation is

$\left|\begin{array}{cc}0-\lambda & 1 \\ -k_{1}{ }^{2} & 0-\lambda\end{array}\right|=0$, where $\lambda$ is a scalar.

Solving, we get

$\lambda^{2}+k_{1}^{2}=0$

Or

$\lambda= \pm i k_{1} \ldots(6)$

Now the characteristic vector for $\lambda=i k_{1}$ is given by

$$
\left[\begin{array}{cc}
0-i k_{1} & 1 \\
-k_{1}{ }^{2} & 0-i k_{1}
\end{array}\right]\left[\begin{array}{l}
y_{1} \\
y_{2}
\end{array}\right]=\left[\begin{array}{l}
0 \\
0
\end{array}\right]
$$

Applying $\mathrm{R}_{2} \rightarrow \mathrm{R}_{2}+i k_{1} \mathrm{R}_{1}$, we can write

This results

$$
\left[\begin{array}{cc}
-i k_{1} & 1 \\
0 & 0
\end{array}\right]\left[\begin{array}{l}
y_{1} \\
y_{2}
\end{array}\right]=\left[\begin{array}{l}
0 \\
0
\end{array}\right]
$$

$-i k_{1} y_{1}+y_{2}=0$

Or

$\left[\begin{array}{l}y_{1} \\ y_{2}\end{array}\right]=\left[\begin{array}{c}1 \\ i k_{1}\end{array}\right]$

The characteristic vector for $\lambda=-i k_{1}$ is given by

$$
\left[\begin{array}{cc}
0+i k_{1} & 1 \\
-k_{1}{ }^{2} & 0+i k_{1}
\end{array}\right]\left[\begin{array}{l}
y_{1} \\
y_{2}
\end{array}\right]=\left[\begin{array}{l}
0 \\
0
\end{array}\right]
$$

Applying $\mathrm{R}_{2} \rightarrow \mathrm{R}_{2}-i k_{1} \mathrm{R}_{1}$, we can write

This results

$$
\left[\begin{array}{cc}
i k_{1} & 1 \\
0 & 0
\end{array}\right]\left[\begin{array}{l}
y_{1} \\
y_{2}
\end{array}\right]=\left[\begin{array}{l}
0 \\
0
\end{array}\right]
$$

$i k_{1} y_{1}+y_{2}=0$

Or

$\left[\begin{array}{l}y_{1} \\ y_{2}\end{array}\right]=\left[\begin{array}{c}1 \\ -i k_{1}\end{array}\right]$

The modal matrix of characteristic vectors is $\left[\begin{array}{cc}1 & 1 \\ i k_{1} & -i k_{1}\end{array}\right]$. Let $\mathrm{P}=\left[\begin{array}{cc}1 & 1 \\ i k_{1} & -i k_{1}\end{array}\right]$, then the inverse of modal matrix $\mathrm{P}$ is given by

$\mathrm{P}^{-1}=\left[\begin{array}{cc}\frac{1}{2} & \frac{1}{2 i k_{1}} \\ \frac{1}{2} & \frac{-1}{2 i k_{1}}\end{array}\right] \ldots \ldots$

To find $P e^{\lambda y} P^{-1}$,
$P e^{\lambda y} P^{-1}=\left[\begin{array}{cc}1 & 1 \\ i k_{1} & -i k_{1}\end{array}\right]\left[\begin{array}{cc}e^{i k_{1} y} & 0 \\ 0 & e^{-i k_{1} y}\end{array}\right]\left[\begin{array}{cc}\frac{1}{2} & \frac{1}{2 i k_{1}} \\ \frac{1}{2} & \frac{-1}{2 i k_{1}}\end{array}\right]$

$=\left[\begin{array}{cc}e^{i k_{1} y} & e^{-i k_{1} y} \\ i k_{1} e^{i k_{1} y} & -i k_{1} e^{-i k_{1} y}\end{array}\right]\left[\begin{array}{cc}\frac{1}{2} & \frac{1}{2 i k_{1}} \\ \frac{1}{2} & \frac{-1}{2 i k_{1}}\end{array}\right]$

$=\left[\begin{array}{cc}\frac{1}{2}\left(e^{i k_{1} y}+e^{-i k_{1} y}\right) & \frac{1}{2 i k_{1}}\left(e^{i k_{1} y}-e^{-i k_{1} y}\right) \\ \frac{i k_{1}}{2}\left(e^{i k_{1} y}-e^{-i k_{1} y}\right) & \frac{1}{2}\left(e^{i k_{1} y}+e^{-i k_{1} y}\right)\end{array}\right]$

$=\left[\begin{array}{cc}\cos _{1} y & \frac{1}{k_{1}} \sin k_{1} y \\ -k_{1} \sin k_{1} y & \cos k_{1} y\end{array}\right]$

Applying $\varphi_{2_{1}}(0)=0$ and $D_{y} \varphi_{2}(0)=\mathrm{b}$, we can write

$\left[\begin{array}{l}\varphi_{21}(y) \\ \varphi_{2_{2}}(y)\end{array}\right]=\left[\begin{array}{cc}\cos _{1} y & \frac{1}{k_{1}} \sin _{1} y \\ -k_{1} \sin k_{1} y & \cos _{1} y\end{array}\right]\left[\begin{array}{l}0 \\ b\end{array}\right]$

$\mathrm{Or}$

$\left[\begin{array}{l}\varphi_{21}(y) \\ \varphi_{22}(y)\end{array}\right]=\left[\begin{array}{l}\frac{b}{k_{1}} \sin k_{1} y \\ b \cos k_{1} y\end{array}\right]$

This gives

$\varphi_{2}(y)=\varphi_{21}(y)=\frac{b}{k_{1}} \sin \left(k_{1} \mathrm{y}\right)$

To solve (3) by Matrix method [5-7],

Let $\varphi_{3}(y)=\varphi_{3_{1}}(y)$

And

$\varphi_{31}{ }^{\prime}(y)=\varphi_{3_{2}}(y) \ldots(14)$

We can rewrite (3) as

$\varphi_{32}{ }^{\prime}(y)-k_{2}^{2} \varphi_{3_{1}}(y)=0$

Or

$\varphi_{32}{ }^{\prime}(y)={k_{2}}^{2} \varphi_{3{ }_{1}}(y) \ldots .(15)$

Equations (18) and (19) can be written in matrix form as $\left[\begin{array}{l}\varphi_{31}{ }^{\prime}(y) \\ \varphi_{32}{ }^{\prime}(y)\end{array}\right]=\left[\begin{array}{cc}0 & 1 \\ k_{2}{ }^{2} & 0\end{array}\right]\left[\begin{array}{l}\varphi_{3_{1}}(y) \\ \varphi_{3_{2}}(y)\end{array}\right]$

The characteristic equation is

$\left|\begin{array}{cc}0-\lambda & 1 \\ k_{2}{ }^{2} & 0-\lambda\end{array}\right|=0$

Solving, we get

$\lambda^{2}-k_{2}^{2}=0$

Or

$\lambda= \pm k_{2} \ldots$ (17)

Now the characteristic vector for $\lambda=i k_{2}$ is given by

$\left[\begin{array}{cc}0-k_{2} & 1 \\ k_{2}{ }^{2} & 0-k_{2}\end{array}\right]\left[\begin{array}{l}y_{1} \\ y_{2}\end{array}\right]=\left[\begin{array}{l}0 \\ 0\end{array}\right]$

Applying $\mathrm{R}_{2} \rightarrow \mathrm{R}_{2}+k_{2} \mathrm{R}_{1}$, we can write

$\left[\begin{array}{cc}-k_{2} & 1 \\ 0 & 0\end{array}\right]\left[\begin{array}{l}y_{1} \\ y_{2}\end{array}\right]=\left[\begin{array}{l}0 \\ 0\end{array}\right]$

This results

$-k_{2} y_{1}+y_{2}=0$

Or

$\left[\begin{array}{l}y_{1} \\ y_{2}\end{array}\right]=\left[\begin{array}{c}1 \\ k_{2}\end{array}\right]$ 
The characteristic vector for $\lambda=-i k_{2}$ is given by

$\left[\begin{array}{cc}0+k_{2} & 1 \\ k_{2}{ }^{2} & 0+k_{2}\end{array}\right]\left[\begin{array}{l}y_{1} \\ y_{2}\end{array}\right]=\left[\begin{array}{l}0 \\ 0\end{array}\right]$

Applying $\mathrm{R}_{2} \rightarrow \mathrm{R}_{2}-k_{2} \mathrm{R}_{1}$, we can write

This results

$$
\left[\begin{array}{cc}
k_{2} & 1 \\
0 & 0
\end{array}\right]\left[\begin{array}{l}
y_{1} \\
y_{2}
\end{array}\right]=\left[\begin{array}{l}
0 \\
0
\end{array}\right]
$$

$k_{2} y_{1}+y_{2}=0$

Or

$\left[\begin{array}{l}y_{1} \\ y_{2}\end{array}\right]=\left[\begin{array}{c}1 \\ -k_{2}\end{array}\right]$

The modal matrix of characteristic vectors is $\left[\begin{array}{cc}1 & 1 \\ k_{2} & -k_{2}\end{array}\right]$. Let $\mathrm{P}=\left[\begin{array}{cc}1 & 1 \\ k_{2} & -k_{2}\end{array}\right]$, then the inverse modal matrix $\mathrm{P}$ is given by

$\mathrm{P}^{-1}=\left[\begin{array}{cc}\frac{1}{2} & \frac{1}{2 k_{2}} \\ \frac{1}{2} & \frac{-1}{2 k_{2}}\end{array}\right] \ldots$

To find $P e^{\lambda y} P^{-1}$,

$P e^{\lambda y} P^{-1}=\left[\begin{array}{cc}1 & 1 \\ k_{2} & -k_{2}\end{array}\right]\left[\begin{array}{cc}e^{k_{2} y} & 0 \\ 0 & e^{-k_{2} y}\end{array}\right]\left[\begin{array}{cc}\frac{1}{2} & \frac{1}{2 k_{2}} \\ \frac{1}{2} & \frac{-1}{2 k_{2}}\end{array}\right]$

$=\left[\begin{array}{cc}e^{k_{2} y} & e^{-k_{2} y} \\ k_{2} e^{k_{2} y} & -k_{2} e^{-k_{2} y}\end{array}\right]\left[\begin{array}{cc}\frac{1}{2} & \frac{1}{2 k_{2}} \\ \frac{1}{2} & \frac{-1}{2 k_{2}}\end{array}\right]$

$=\left[\begin{array}{cc}\frac{1}{2}\left(e^{k_{2} y}+e^{-k_{2} y}\right) & \frac{1}{2 k_{2}}\left(e^{k_{2} y}-e^{-k_{2} y}\right) \\ \frac{k_{2}}{2}\left(e^{k_{2} y}-e^{-k_{2} y}\right) & \frac{1}{2}\left(e^{k_{2} y}+e^{-k_{2} y}\right)\end{array}\right]$

$=\left[\begin{array}{cc}\cosh _{2} y & \frac{1}{k_{2}} \sinh _{2} y \\ k_{2} \sinh _{2} y & \cosh _{2} y\end{array}\right]$.

Applying $\varphi_{3_{1}}(0)=a$ and $D_{y} \varphi_{3}(0)=\mathrm{b}$, we can write

Or

$$
\left[\begin{array}{l}
\varphi_{\mathrm{R}_{1}}(y) \\
\varphi_{\mathrm{R}_{2}}(y)
\end{array}\right]=\left[\begin{array}{cc}
\cos _{2} y & \frac{1}{k_{2}} \sin _{2} y \\
k_{2} \sin k_{2} y & \cos _{2} y
\end{array}\right]\left[\begin{array}{l}
a \\
b
\end{array}\right]
$$

Or

$$
\left[\begin{array}{l}
\varphi_{3_{1}}(y) \\
\varphi_{3_{2}}(y)
\end{array}\right]=\left[\begin{array}{c}
a \cos k_{2} y+\frac{b}{k_{2}} \sin _{2} y \\
a k_{2} \sin k_{2} y+b \cos k_{2} y
\end{array}\right]
$$

$\varphi_{3}(y)=\varphi_{3_{1}}(y)=a \cosh k_{2} y+\frac{b}{k_{2}} \sinh k_{2} y$.

Or

$\varphi_{3}(\mathrm{y})=a \frac{e^{k_{2} y}+e^{-k_{2} y}}{2}+\frac{b}{k_{2}} \frac{e^{k_{2} y}-e^{-k_{2} y}}{2}$

Or

$\varphi_{3}(\mathrm{y})=\left(\frac{a}{2}+\frac{b}{2 k_{2}}\right) e^{k_{2} y}+\left(\frac{a}{2}-\frac{b}{2 k_{2}}\right) e^{-k_{2} y}$

Now $\varphi_{3}(\mathrm{y})$ approaches to zero at $y$

$$
=\text { infinity only if }
$$

$\left(\frac{a}{2}+\frac{b}{2 k_{2}}\right)=0$

Or

$b=-k_{2} a \ldots .(27)$

Using (27) in (26), we can write

$\varphi_{3}(\mathrm{y})=a e^{-k_{2} y}$

Applying $\varphi_{2}(\mathrm{~L})=\varphi_{3}(\mathrm{~L})$ and solving, we get

$\frac{b}{k_{1}} \sin \left(k_{1} \mathrm{~L}\right)=a e^{-k_{2} L}$

Or

$\mathrm{a}=\frac{b}{k_{1}} \sin \left(k_{1} \mathrm{~L}\right) e^{k_{2} L}$

Using (29) in (28). We get

$\varphi_{3}(\mathrm{y})=\frac{b}{k_{1}} \sin \left(k_{1} \mathrm{~L}\right) e^{k_{2} L} e^{-k_{2} y}$

Or

$\varphi_{3}(\mathrm{y})=\frac{b}{k_{1}} \sin \left(k_{1} \mathrm{~L}\right) e^{-k_{2}(y-L)}$.

According to the normalization condition $[5,9]$, we have

$$
\begin{aligned}
& \int_{\substack{y=-\infty \\
\text { Or }}}^{y=\infty} \varphi(\mathrm{y}) \varphi(\mathrm{y})^{*} \mathrm{dy}=1 \\
& \quad \int_{y=0}^{y=L} \varphi_{2}(\mathrm{y}) \varphi_{2}(\mathrm{y}) * \mathrm{dy}+\int_{y=L}^{y=\infty} \varphi_{3}(\mathrm{y}) \varphi_{3}(\mathrm{y}) * \mathrm{dy}=1
\end{aligned}
$$

Using (12) and (30) and simplifying, we get

$\mathrm{b}=\frac{k_{1}}{\sqrt{\frac{L}{2}+\frac{\sin \left(k_{1} \mathrm{~L}\right)}{4 k_{1} L}+\frac{\sin ^{2}\left(k_{2} \mathrm{~L}\right)}{2 k_{2} L}}} \ldots \ldots$

Again, applying $\varphi_{2}{ }^{\prime}(\mathrm{L})=\varphi_{3}{ }^{\prime}(\mathrm{L})$ and solving, we get $\tan \left(k_{1} \mathrm{~L}\right)=-k_{1} k_{2} \ldots . .(32)$

This is the required transcendental equation determining the discrete eigenvalues for bound state.

The corresponding eigenwave function is given by

$\varphi(\mathrm{y})=$
$\left[\begin{array}{c}\frac{1}{\sqrt{\frac{L}{2}+\frac{\sin \left(k_{1} \mathrm{~L}\right)}{4 k_{1} L}+\frac{\sin ^{2}\left(k_{2} \mathrm{~L}\right)}{2 k_{2} L}}} \operatorname{sor} \mathrm{y}<0 \\ \frac{1}{\sqrt{\frac{L}{2}+\frac{\sin \left(k_{1} \mathrm{~L}\right)}{4 k_{1} L}+\frac{\sin ^{2}\left(k_{2} \mathrm{~L}\right)}{2 k_{2} L}}} \sin \left(k_{1} \mathrm{y}\right) \text { for } 0<y<L\end{array}\right.$.

\section{Conclusion}

The time-independent Schrodinger's equation for halfinfinite potential well has been solved successfully by matrix method to find the transcendental equation determining the discrete eigenvalues for bound state and 
the corresponding eigenwave function. The results obtained are the same as obtained with ordinary algebraic and analytical methods [1-3, 13, 14]. The matrix method has been proved to be a successful approach for solving the half-infinite potential well problem.

\section{References}

[1] N. Zettili, "Quantum Mechanics; Concepts and Applications".Publisher: Wiley India Pvt. Ltd.

[2] J. Griffiths, "Introduction to Quantum Mechanics". $2^{\text {nd }}$ edition. Publisher: Cambridge University Press, 2017.

[3] B.N. Srivastava, "Quantum Mechanics". 16 ${ }^{\text {th }}$ edition, 2017 Publisher, Pragati Prakashan, 1980.

[4] H. K. Dass, 'Advanced Engineering Mathematics', 2014. Publisher: S. Chand Publications.

[5] Rohit Gupta, Rahul Gupta, Matrix Method For Solving The Schrodinger's Time - Independent Equation To Obtain The Eigen Functions And Eigen Energy Values of A Particle Inside The Infinite Square Well Potential, IOSR Journal of Applied Physics (IOSR-JAP), Volume 10, Issue 5 Ver. I (Sep. - Oct. 2018), PP. 0105.

[6] Rohit Gupta, Rahul Gupta, "Matrix method approach for the temperature distribution and heat flow along a conducting bar connected between two heat sources", Journal of Emerging Technologies and Innovative Research, Volume 5 Issue 9, September 2018, PP. 210-214.
[7] Rohit Gupta, Rahul Gupta, "Matrix method for deriving the response of a series L- C- R network connected to an excitation voltage source of constant potential", Pramana Research Journal, Volume 8 , Issue 10, 2018.

[8] Rohit Gupta, Rahul Gupta, Sonica Rajput "Response of a parallel ŁC- $\mathcal{R}$ network connected to an excitation source providing a constant current by matrix method", International Journal for Research in Engineering Application \& Management (IJREAM), Vol-04, Issue07, Oct 2018.

[9] Rohit Gupta, Tarun Singhal, Dinesh Verma, Quantum mechanical reflection and transmission coefficients for a particle through a onedimensional vertical step potential, International Journal of Innovative Technology and Exploring Engineering, Volume-8, Issue-11, September 2019, PP 2882-2886.

[10] Rohit Gupta, Yuvraj Singh Chib, Rahul Gupta, Design of the resistor-capacitor snubber network for a d. c. circuit containing an inductive load, Journal of Emerging Technologies and Innovative Research (JETIR), Volume 5, Issue 11, November 2018, pp. 68-71.

[11] Rohit Gupta, Rahul Gupta, Sonica Rajput, Analysis of Damped Harmonic Oscillator by Matrix Method, International Journal of Research and Analytical Reviews (IJRAR), Volume 5, Issue 4, October 2018, pp. 479-484.

[12] Rohit Gupta, Rahul Gupta, Heat Dissipation From The Finite Fin Surface Losing Heat At The Tip, International Journal of Research and Analytical Reviews, Volume 5, Issue 3, September 2018, pp. 138-143.

[13] P.A.M. Dirac, 'Principles of quantum mechanics'. Reprint 2016. Publisher: Snowball Publishing (2012).

[14] P. M. Mathews, 'A Textbook of Quantum Mechanics'. Publisher: Tata McGraw Hill Education Private Limited, 2010. 